Ann. Biol. anim. Bioch. Biophys., I97I, 11 (I), 69-84.

\title{
INFLUENCE DE LA SUPPLEMENTATION PROTÉTQUE BI-HEBDOMADAIRE D'UN REGIME A BAS NIVEAU AZOTÉ SUR LE GAIN DE POIDS ET LES BILANS D'AZOTE CHEZ LE PORG EN CROISSANCE
}

\author{
Genevièvre CHARLET-LERY \\ avec la collaboration technique de Michèle Fiszlewicz, Marie-Thérèse Morel \\ et S. Guéneau \\ Laboratoire de Recherches sur la Conservation et l'Efficacité des Aliments, \\ Centre national de Recherches zootechniques, I. N.R. A., \\ 78 - Jouy-en-Josas
}

\section{RÉSUMÉ}

Chez le Porc en croissance $(25-97 \mathrm{~kg}$ ), la régularité de l'apport protéique à tous les repas (alimentation classique) ou sa concentration partielle mais importante (40 p. Ioo en 2 repas riches en azote (HN) sur les 13 hebdomadaires (alimentation dissociée)) ne modifient ni la croissance, ni les indices de consommation, ni les bilans d'azote mesurés $2 \mathrm{I}$ jours consécutifs aux environs de $40 \mathrm{~kg}$ puis de $70 \mathrm{~kg}$.

Les carcasses des animaux soumis à l'alimentation dissociée auraient tendance à être légèrement moins adipeuses.

On suppose que l'organisme animal stockerait momentanément les protéines qui lui sont fournies irrégulièrement et les utiliserait dans les deux jours suivants.

\section{INTRODUCTION}

Deux expériences précédentes effectuées sur des porcs en croissance nous ont conduits aux résultats suivants :

Io Contrairement aux observations classiques faites chez le Rat ou le Chien adulte, nous observons (CHARLET-LERY, 1970) chez le Porc en croissance (30-60 kg) à la suite de la consommation d'un repas à haut taux azoté (HN)des dépenses d'extrachaleur inférieures à celles induites par les repas à taux azoté bas ou moyen (BN) qu'il ingère régulièrement. L'économie d'énergie par gramme d'azote ingéré en plus au cours du repas $\mathrm{HN}\left(\mathrm{g} \mathrm{N}_{\mathrm{i}}+\right.$ ) s'élève à $2,34 \mathrm{kcal}$ dans nos conditions expérimen- 
tales : source protéique de haute valeur biologique identique pour les 2 types de repas, g $N_{1}+$ par unité de poids métabolique $\left(\mathrm{P}^{0,70}\right)$ variant de $2, \mathrm{I} 6$ à 4,82 .

Cette économie s'accompagne d'une chute des quotients respiratoires relativement constante de $I_{5}$ à $20 \mathrm{p}$. Ioo, les faisant passer de valeurs supérieures à I à des valeurs inférieures à $\mathrm{x}$. Ceci montre, qu'il y a, pendant les 7 heures qui suivent le repas, non seulement un arrêt total de la lipogenèse, mais peut-être aussi lipolyse puisque certains $Q R$ se rapprochent de 0,7 .

Sur ces mêmes animaux, nous avons constaté que l'azote apporté par le repas HN n'est pas éliminé brutalement : dans les 24 heures qui suivent le repas, de 30 à 80 p. Ioo de $\mathrm{N}_{i}+$ restent apparemment retenus par l'organisme.

$2^{\circ}$ Chez les animaux dont tous les repas sont réguliers en quantité et qualité, il existe une corrélation positive et hautıment significative entre le métabolisme de base approché (M. Ba) ou métabolisme d'entretien et la quantité d'azote ingéré( $\left.\mathrm{N}_{\mathrm{i}}\right)$ journellement par 1'animal (CHARLET-LERY, I960), selon une relation linéaire.

$$
r=+0,74 \pm 0,08
$$

L'étude des corrélations multiples montre que la quantité de matière sèche ingérée joue un rôle de beaucoup plus faible importance que celui joué par les protéines et qu'il n'y a pas interférence du poids de l'animal, dans la limite de nos conditions expérimentales (porc de 25 à $78 \mathrm{~kg}$ ingérant par unité de poids métabolique $\left(\mathrm{P}^{0,70}\right)$ de 75,2 à $\mathrm{I} 45,4 \mathrm{~g}$ de MS et de 0,03 à $\left.5,8 \mathrm{~g} \mathrm{~N}\right)$.

La confrontation de ces deux séries de faits nous a suggéré 1'hypothèse suivante : dans la limite où les protéines du repas $\mathrm{HN}$ malgré leur apport massif et brutal sont utilisées par l'animal, la distribution régulière d'un régime à taux azoté bas complété par quelques repas à taux azoté élevé, c'est-à-dire la dissociation partielle des consommations d'énergie et d'azote devrait :

$I^{\circ}$ ne pas entraîner des dépenses énergétiques plus élevées qu'un régime normal mais peut-être même pourrait provoquer une légère diminution des dépenses caloriques (abaissement du métabolisme d'entretien et faibles dépenses d'extra-chaleur après les repas azotés) ;

$2^{\circ}$ provoquer une moindre adiposité des carcasses par suite de la suppression de la lipogenèse consécutivement aux repas $H N$. Une telle dissociation chez le Porc en croissance pourrait être une méthode d'alimentation susceptible de fournir des carcasses plus maigres.

C'est ce que nous avons tenté de vérifier en comparant les bilans d'azote, les gains de poids, 1'indice de consommation, la qualité des carcasses chez 2 lots de porcs entre 25 et $95 \mathrm{~kg}$, nourris selon les deux méthodes d'alimentation classique (C) ou dissociée (D). Les mêmes quantités d'énergie et de protéines sont apportées hebdomadairement aux animaux, soit par des repas tous identiques chez ceux soumis au régime classique, soit par des repas à bas taux azoté (BN) et à taux azoté haut (HN) chez ceux soumis au régime dissocié. Mais afin de limiter rigoureusement nos observations a la dissociation énergie-protéines, les autres besoins des animaux ont été régulièrement couverts à tous les repas (matières minérales, vitamines).

Un essai précédent de croissance (CHARLET-LERY, I967) en alimentation sèche avait montré l'inappétibilité des agglomérés $H N$, ce qui n'avait pas permis l'égalité des ingérés protéiques, même en redistribuant les refus $H N$ au repas du soir. Aussi, dans l'expérience actuelle, l'alimentation est distribuée sous forme semi-liquide. 


\section{MATÉRIEL ET MÉTHODES}

\section{Les animaux}

Douze porcs mâles castrés de race Large White pesant aux environs de $25 \mathrm{~kg}$ couplés par groupe de 2 frères, constituent deux groupes homogènes. Ils sont logés au sol dans des cases identiques permettant le contrôle individuel des rations ingérées.

Au cours de la croissance entre 30 et $45 \mathrm{~kg}$ puis entre 60 et $85 \mathrm{~kg}$, on effectue les mesures de bilan sur 3 couples d'animaux, soit 3 par lot, placés en cage à métabolisme. La température des salles est maintenue aux environs de $\mathbf{r}^{\circ}$. Les animaux sont pesés hebdomadairement.

\section{Les régimes}

Les animaux mangent 2 repas par jour sauf le dimanche soir. Ainsi, l'identité des apports nutritifs s'effectue sur les $\mathrm{I}_{3}$ repas distribués par semaine.

Pour assurer cette identité entre les deux groupes d'animaux, les mêmes mélanges alimentaires BN et HN sont utilisés pour tous les 2. Le mélange BN constitue les I I repas BN et le mélange HN les 2 repas HN des animaux soumis au régime dissocié. Les I $3_{3}$ repas des animaux soumis au régime classique sont composés de ces deux mélanges dans le rapport II-2. Le rythme (I I-2) a été choisi car il correspondait à celui de nos études sur l'extra-chaleur. Les repas HN sont distribués le matin à jour fixe. Leur espacement est alternativement de 3 jours $(72 \mathrm{~h})$ et de 4 jours $(96 \mathrm{~h})$.

La composition des mélanges $\mathrm{BN}$ et HN est calculée afin que le régime ingéré par les animaux apporte I $20 \mathrm{~g}$ de matières azotées digestibles à l'UF. avant $50 \mathrm{~kg}$ et $100 \mathrm{~g}$ après $50 \mathrm{~kg}$. Les taux de matière grasse et de cellulose Weende sont très voisins. Deux mélanges BN sont prévus, l'un avant, l'autre après $50 \mathrm{~kg}$, complétés par un seul mélange HN qui sert durant toute l'expérience (tabl. I). On peut donc calculer que les deux repas HN apportent, au cours du bilan I, 37 p. 100 et, au cours du bilan II, 43 p. Ioo des protéines ingérées dans la semaine.

TABLEAU I

Composition des mélanges alimentaires

\begin{tabular}{|c|c|c|c|}
\hline & \multicolumn{2}{|c|}{ Mélanges BN } & \multirow{2}{*}{$\begin{array}{c}\text { Mélange } \\
\text { HN }\end{array}$} \\
\hline & Avant $50 \mathrm{~kg}$ & Après $50 \mathrm{~kg}$ & \\
\hline & \multicolumn{3}{|c|}{ En \% brut } \\
\hline Orge $\ldots \ldots \ldots \ldots \ldots \ldots \ldots \ldots$ & 20 & 10 & 14 \\
\hline Maïs $\ldots \ldots \ldots \ldots \ldots \ldots \ldots \ldots$ & 15 & 25 & 14 \\
\hline 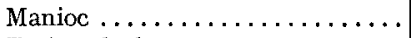 & 45 & 50 & - \\
\hline Farine de luzerne $\ldots \ldots \ldots \ldots$ & 10 & 8 & 12 \\
\hline Farine de poisson $\ldots \ldots \ldots \ldots \ldots$ & 7 & 4 & 24 \\
\hline Tourteau de soja $\ldots \ldots \ldots \ldots$ & - & $\longrightarrow$ & 34 \\
\hline \multirow[t]{2}{*}{ Complément minéral vitaminé .. } & 3 & $\mathbf{3}$ & 2 \\
\hline & \multicolumn{3}{|c|}{ En $\%$ sec } \\
\hline Matières minérales $\ldots \ldots \ldots \ldots$. & 6,94 & 6,28 & 8,73 \\
\hline Matière organique $\ldots \ldots \ldots \ldots$ & 93,06 & 93,72 & 91,27 \\
\hline Azote $\ldots \ldots \ldots \ldots \ldots \ldots \ldots$ & 1,98 & 1,52 & 6,37 \\
\hline Matières azotées $(N \times 6,25) \ldots$ & 12,37 & 9,50 & 39,81 \\
\hline Cellulose Weende...$\ldots \ldots \ldots$ & 5,62 & 5,64 & 5,45 \\
\hline Matières grasses $\ldots \ldots \ldots \ldots \ldots$ & 2,28 & 2,01 & 3,57 \\
\hline Extractif non azoté.......... & 72,79 & 76,57 & 42,44 \\
\hline
\end{tabular}


La quantité de matière sèche distribuée chaque jour en 2 repas égaux s'élève à 3,80 p. Ioo du poids vif de chaque animal jusqu'à $65 \mathrm{~kg}$ et à 3,50 après $65 \mathrm{~kg}$. Les repas sont distribués à l'état humide (eau/MS $=2,5$ ) et les refus sont pesés après chaque repas.

\section{Les bilans}

Les animaux sont mis en cage trois jours avant le début du bilan sans aucune modification du programme alimentaire. L'irrégularité des apports azotés au cours de la semaine et celle consécutive des émissions urinaires d'azote, que nous avons précédemment vérifiées sur un animal, nécessitent que les bilans soient exécutés durant 7 jours consécutifs. Mais ignorant les possibilités đe variations du phénomène que nous cherchions à étudier, nous avons décidé de multiplier nos mesures : les bilans sont répétés 3 semaines consécutives. Par semaine, il est constitué en vue des analyses, des échantillons proportionnels, un pour les aliments, deux pour les fèces (I sur 3 jours, I sur 4 jours) et trois pour l'urine ( 2 sur 2 jours et I sur 3 jours). L'acide sulfurique utilisé pour la conservation de cette dernière est mise dans les bidons de collecte. Les échantillons sont gardés à $2^{\circ}$ les analyses d'azote sont faites par micro-Kjeldahl.

Le dosage de la matière sèche des matières fécales est effectué tous les jours. L'échantillon sec cumulé par période de 7 jours sert à l'analyse des matières minérales et cellulosiques.

Les divers coefficients sont calculés pour chaque semaine.

\section{Fréquence respiratoire}

L'étude menée parallèlement des paramètres physiologiques susceptibles d'être modifiés par la richesse en protéines des repas, a montré que l'abaissement des dépenses énergétiques postprandiales après le repas HN, s'accompagnait d'une chute des fréquences respiratoires (données non publiées). Cette mesure particulièrement simple à effectuer sur les animaux en cage à bilan, a été choisie comme test reflétant les variations du métabolisme énergétique.

\section{Abattage}

En fin d'expérience, les animaux sont abattus au poids le plus proche de roo $\mathrm{kg}$. Les carcasses sont préparées selon la découpe parisienne et classés suivant la grille commerciale correspondante (ITP I964). Cette méthode a été choisie plutôt que l'analyse des carcasses plus coûteuse et plus laborieuse, à cause de sa simplicité mais elle n'est évidemment qu'une simple méthode corrélative de composition.

\section{RÉSULTATS}

Deux animaux ont été malades successivement en fin d'expérience (couple 4-10), ce qui nous a conduits à supprimer ces animaux de l'essai croissance. Cependant, ils ont participé au premier bilan et leurs résultats ne se différenciant pas de ceux des autres sujets, ont été mentionnés.

\section{Les bilans}

Les résultats moyens journaliers sont portés dans les tableaux 2,3 et 4 .

\section{Niveaux de consommation.}

Tant au cours du bilan I qu'au cours du bilan II, les niveaux moyens journaliers de consommation sont très proches, pour les 2 lots, qu'ils soient exprimés en valeur absolue ou rapportés au poids métabolique $\left(\mathrm{MS}_{\mathrm{i}} / \mathrm{P}^{0,75}:\right.$ 90,20 et 90,00 (I) 97,9 et $99, \mathrm{I}$ (II) - $\mathrm{N}_{1} / \mathrm{P}^{0,75}: 2,34$ et 2,35 (I) 2,22 et 2,22 (II)). Cette homogénéité, due à l'absence presque complète de refus, nous assure que les animaux se sont tous trouvés dans des conditions alimentaires parfaitement comparables. 
TABLEAU 2

Résultats moyens journaliers

\begin{tabular}{c|c|c|c|c|c|c|c|c}
\hline \hline \multirow{3}{*}{ Animal } & $\begin{array}{c}\text { Poids } \\
(\mathrm{kg})\end{array}$ & $\begin{array}{c}\text { Croissance } \\
(\mathrm{g} / \mathrm{j})\end{array}$ & $\begin{array}{c}\mathrm{MS}_{\mathrm{i}} \\
(\mathrm{g})\end{array}$ & $\begin{array}{c}\mathrm{MO}_{\mathrm{d}} \\
(\mathrm{g})\end{array}$ & $\begin{array}{c}\mathrm{N}_{\mathrm{i}} \\
(\mathrm{g})\end{array}$ & $\begin{array}{c}\mathrm{N}_{\mathbf{1}} \\
(\mathrm{g})\end{array}$ & $\begin{array}{c}\mathrm{N}_{\mathrm{u}} \\
(\mathrm{g})\end{array}$ & $\begin{array}{c}\mathrm{N} \text { bilan } \\
(\mathrm{g})\end{array}$ \\
\hline
\end{tabular}

\section{Alimentation classique}

\begin{tabular}{c|l|l|l|}
3 & 37,3 & 485 & 1336 \\
4 & 41,7 & 690 & 1495 \\
5 & 39,4 & 450 & 1426 \\
Moyenne & 39,5 & 542 & 1419
\end{tabular}

\begin{tabular}{c|c|c|c}
3 & 74,9 & 990 & 2524 \\
5 & 71,4 & 820 & 2366 \\
Moyenne & 73,2 & 905 & 2445
\end{tabular}

Alimentation dissocièe

\begin{tabular}{|c|c|c|c|c|c|c|c|c|}
\hline 9 & 37,6 & 510 & 1341 & 1017 & 35,2 & 7,4 & 9,7 & 18,1 \\
\hline 10 & 43,0 & 650 & 1509 & 1133 & 39,4 & 9,3 & 14,0 & 16,1 \\
\hline 11 & 42,0 & 565 & 1503 & 1152 & 39,5 & 10,0 & 11,3 & 18,2 \\
\hline Moyenne & 40,9 & 575 & 1451 & 1101 & 38,0 & & & 17,5 \\
\hline \multicolumn{9}{|c|}{ Bilan II } \\
\hline 9 & 73,7 & 930 & 2469 & 1997 & 56,6 & 12,5 & 17,9 & 26,2 \\
\hline 11 & 77,5 & 930 & 2603 & 2103 & 59,7 & 12,9 & 21,9 & 24,9 \\
\hline Moyenne & 75,6 & 930 & 2536 & 2050 & 58,2 & & & 25,5 \\
\hline
\end{tabular}

Digestibilité des matières sèches, organiques et cellulosiques.

Les coefficients de digestibilité hebdomadaire des matières sèches et organiques (CUD MS-CUD MO) sont pour un même animal toujours très proches les uns des autres comme le montrent les valeurs des CUD MS obtenus pour les 6 animaux au cours du bilan I et transcrits à titre indicatif (tabl. 3), avec leurs erreurs types.

TABLEAU 3

Coefficients moyens d'utilisation digestive ( $\pm s m$ )

\begin{tabular}{|c|c|c|c|c|c|c|}
\hline & \multicolumn{3}{|c|}{ Bilan I } & \multicolumn{3}{|c|}{ Bilan II } \\
\hline & $\begin{array}{c}\text { Matière } \\
\text { sèche }\end{array}$ & $\begin{array}{c}\text { Matière } \\
\text { organique }\end{array}$ & $\begin{array}{l}\text { Cellulose } \\
\text { Weende }\end{array}$ & $\begin{array}{l}\text { Matière } \\
\text { sèche }\end{array}$ & $\begin{array}{c}\text { Matière } \\
\text { organique }\end{array}$ & $\begin{array}{l}\text { Cellulose } \\
\text { Weende }\end{array}$ \\
\hline $\begin{array}{l}\text { Régime } \\
\text { classique }\end{array}$ & $\begin{array}{r}80,4 \\
\pm 0,4\end{array}$ & $\begin{array}{r}83,6 \\
\pm 0,4\end{array}$ & $\begin{array}{r}32,1 \\
\pm 1,2\end{array}$ & $\begin{array}{r}80,6 \\
\pm 0,4\end{array}$ & $\begin{array}{r}83,3 \\
\pm \quad 0,3\end{array}$ & $\begin{array}{r}31,3 \\
\pm 1,2\end{array}$ \\
\hline $\begin{array}{l}\text { Régime } \\
\text { dissocié }\end{array}$ & $\begin{array}{r}83,0 \\
\pm 0,6\end{array}$ & $\begin{array}{r}85,5 \\
\pm 0,5\end{array}$ & $\begin{array}{r}35,8 \\
\pm 1,3\end{array}$ & $\begin{array}{r}81,8 \\
\pm \quad 0,2\end{array}$ & $\begin{array}{r}84,6 \\
\pm \quad 0,2\end{array}$ & $\begin{array}{r}34,0 \\
\pm 1,1\end{array}$ \\
\hline
\end{tabular}




\section{TABLEAU 4}

Coefficients d'utilisation azotée

Moyennes individuelles et par groupe ( $\pm s m)$

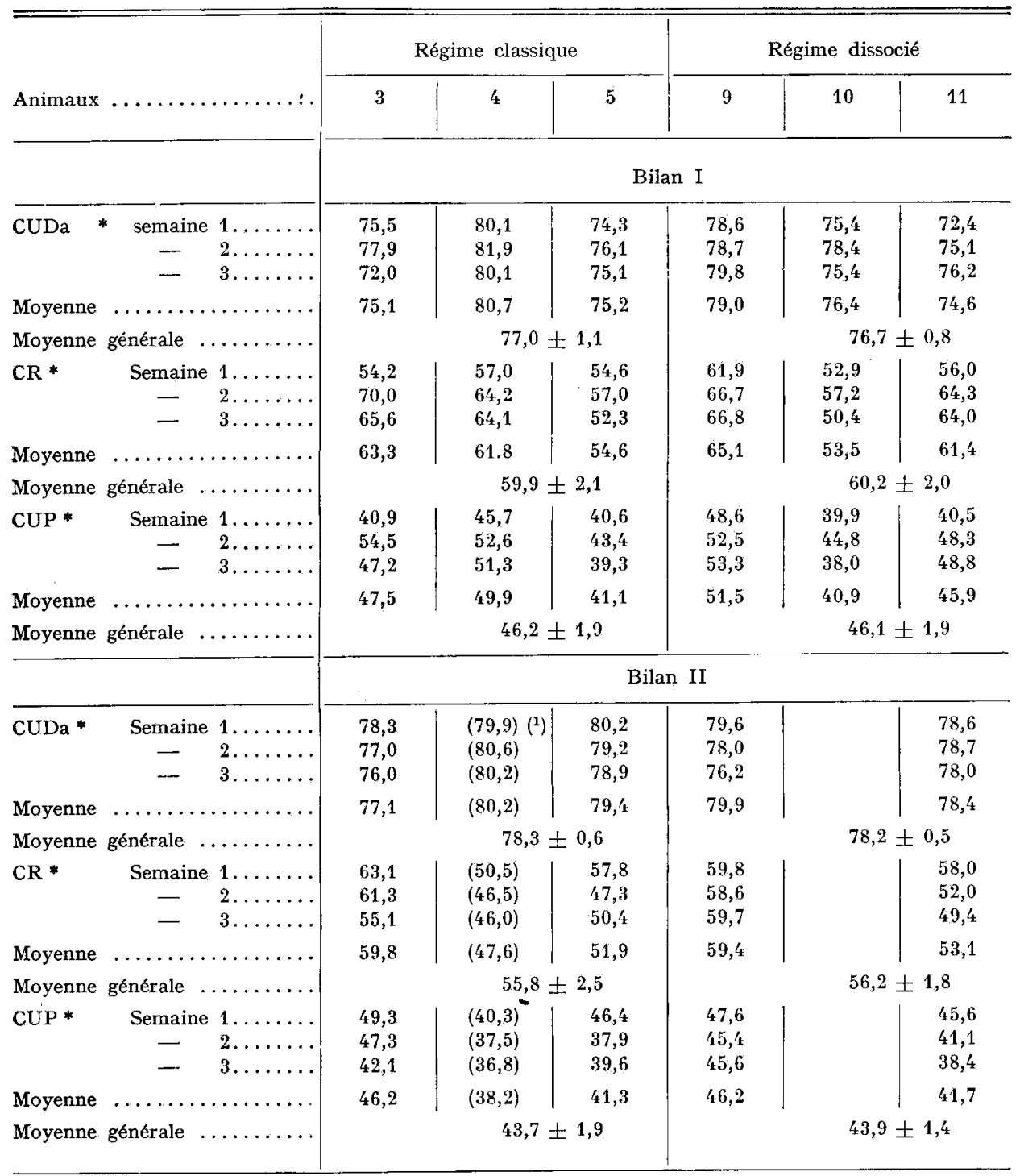

(1) Non inclus dans la moyenne.

* CUDa $=$ Coefficient d'utilisation digestive apparent. $-\mathrm{CR}=$ Coefficient de rétention $\mathrm{I}-\mathrm{F}-\mathrm{U} / \mathrm{I}-\mathrm{F}$. - CUP $=$ Coefficient d'utilisation pratique $\mathrm{I}-\mathrm{F}-\mathrm{U} / \mathrm{I}$. 
Les CUD des matières sèches, organiques et cellulosiques ne diffèrent pas entre les régimes $C$ et $D$ surtout au cours du bilan I effectué avant $50 \mathrm{~kg}$. Après $50 \mathrm{~kg}$, le mélange $\mathrm{BN}$ ayant été mođifié (plus de manioc, moins de farine de luzerne), les CUD sont tous plus élevés qu'au cours du bilan I, d'environ 2 points pour les animaux en régime $\mathrm{C}$ et $\mathrm{I}$ point pour ceux $\mathrm{d} \mathfrak{u}$ régime $\mathrm{D}$. Il existe par conséquent au cours $\mathrm{d} \mathfrak{u}$ bilan II une différence d'environ I point entre les 2 groupes en faveur du régime $\mathrm{C}$, mais cette différence n'est pas significative.

\section{Efficacité des protéines.}

Les données relatives aux protéines sont portées dans le tableau 4 . Il est absolument évident que la distribution de $40 \mathrm{p}$. Ioo des protéines en 2 repas par semaine n'a modifié ni les CUD a : 77,0 et 76,7 (bilan I), 78,3 et 78,2 (bilan II), ni les coefficients de rétention (CR) : 59,9 et 60,2 (bilan I), 55,8 et 56,2 (bilan II), et d'utilisation pratique (CUP) 46,2 et 46,I (bilan I), 43,7 et 43,9 (bilan II), qui restent toujours très proches.

Par contre, on constate entre les deux bilans, un abaissement de quelques points des CR qui passent en moyenne de 60 dans le bilan I à 56 dans le bilan II ce qui entraîne une diminution des CUP de 46 à 44 .

Pour un même animal, et d'une semaine sur l'autre, les CUDa sont très proches les uns des autres. Par contre, les CR subissent des variations beaucoup plus fortes pouvant dépasser $20 \mathrm{p}$. Ioo, ce qui montre l'intérêt d'avoir prolongé l'expérience au delà d'une semaine.

A partir de ces deux séries de données, on calcule que les rations apportent, avant $59 \mathrm{~kg}: \mathrm{I}, 03 \mathrm{UF}$ et I28 $\mathrm{g} \mathrm{MAD} / \mathrm{kg}$ MS soit I24 $\mathrm{g}$ MAD/UF et après $59 \mathrm{~kg}: \mathrm{I}, 04 \mathrm{UF}$ et IIo g MAD/kg MS soit Io6 g MAD/UF.

\section{Croissance et abattage}

La croissance des sujets du lot $\mathrm{D}$ a été toujours très légèrement inférieure à celle du lot $\mathrm{C}$ (tabl. 5). Les gains journaliers de poids vif sont respectivement pour les lots $\mathrm{C}$ et $\mathrm{D}$, entre 26 et $52 \mathrm{~kg}$ de 513 et $502 \mathrm{~g}$, entre 52 et $97 \mathrm{~kg}$ de 839 et $775 \mathrm{~g}$, soit au total entre 26 et $97 \mathrm{~kg}$ de 677 et $644 \mathrm{~g}$. Ceci se répercute sur les indices de consommation plus élevés d'environ $5 \mathrm{p}$. Ioo pour les sujets du lot $\mathrm{D}$

entre 26 et $52 \mathrm{~kg} \quad 2,62$ contre $2,5 \mathrm{I}$

entre 52 et $97 \mathrm{~kg} \quad 3,17$ contre 2,97

entre 26 et $97 \mathrm{~kg} \quad 2,96$ contre $2,8 \mathrm{I}$

TABLEAU 5

Gain journalier et indice de consommation

$\mathrm{g} / \mathrm{j}$ et $\mathrm{kg}$ matière sèche/ $\mathrm{kg}$ de poids vif

\begin{tabular}{|c|c|c|c|c|c|c|}
\hline \multirow{2}{*}{$\begin{array}{l}\text { Limite de poids } \\
(\mathrm{kg})\end{array}$} & \multicolumn{2}{|c|}{26 à 52} & \multicolumn{2}{|c|}{52 à 97} & \multicolumn{2}{|c|}{26 à 97} \\
\hline & Gain & I.C. & Gain & I.C. & Gain & I.C. \\
\hline Lot & & & & & & \\
\hline Classique .. & 513 & $2,51 \pm 0,46$ & 839 & $2,97 \pm 0,27$ & $677 \pm 46$ & $2,81 \pm 0,21$ \\
\hline Dissocié . & 502 & $2,62 \pm 0,57$ & 775 & $3,17 \pm 0,18$ & $644 \pm 7$ & $2,96 \pm \sqrt{0} 0,12$ \\
\hline
\end{tabular}


mais par suite du nombre relativement faible d'animaux, aucune différence n'est significative.

Le tableau 6 indique les résultats d'abattage pour chaque animal. Les rendements sont identiques. Aucune caractéristique ne différencie significativement les 2 groupes. Cependant, les carcasses des animaux dissociés, apparaissent légèrement plus maigres. Le pourcentage de jambon + longe est le plus élevé $(52,4$ contre 51,6$)$, le pourcentage de bardière + panne est le plus faible (I8,5 contre I9,7), de même l'épaisseur de lard $(28,8$ contre 29,5$)$. Le classement est pratiquement identique. Dans le lot témoin, nous avons 2 animaux classés en $B$ et 3 en $C$, dans le lot expérimental 3 en $B$, I en $C$ et $I$ en $D$.

TABLEAU 6

Résultats d'abattage

\begin{tabular}{|c|c|c|c|c|c|c|}
\hline \multirow{2}{*}{ Lot } & \multirow{2}{*}{$\begin{array}{l}\text { Poids vif } \\
\quad(\mathbf{k g})\end{array}$} & \multirow{2}{*}{$\begin{array}{l}\text { Poids net } \\
\quad(k g)\end{array}$} & \multirow{2}{*}{$\begin{array}{c}\text { Rendement } \\
(\%)\end{array}$} & $\underset{\text { longe }}{\text { Jambon }}+$ & $\begin{array}{c}\text { Barde }+ \\
\text { panne }\end{array}$ & \multirow{2}{*}{$\begin{array}{c}\text { Épaisseur } \\
\text { lard dorsal (1) } \\
\text { (mm) }\end{array}$} \\
\hline & & & & \multicolumn{2}{|c|}{$\%$ du poids net } & \\
\hline Classique & 98,6 & 71,2 & 72,2 & $51,6 \pm 2,7$ & $19,7 \pm 3,0$ & $29,5 \pm 4,7$ \\
\hline Dissocié $\ldots . . . \ldots$ & 95,9 & 70,1 & 73,0 & $52,4 \pm 2,2$ & $18,5 \pm 2,3$ & $28,8 \pm 7,3$ \\
\hline
\end{tabular}

(') Rein $+\operatorname{dos} / 2$

Cette moindre adiposité des carcasses en alimentation $\mathrm{D}$ avait été observée en alimentation sèche malgré une croissance ralentie ; en particulier, 1'épaisseur moyenne du lard dorsal était abaissée $(24,5$ contre $25,7 \mathrm{~mm})$.

\section{Fréquence respiratoire}

Les fréquences respiratoires mesurées sur les 2 séries d'animaux, 2, 3 et 4 heures après le repas du matin au cours des bilans I et II, montrent que :

- En alimentation $\mathrm{C}$, les données obtenues sont variables d'un jour sur l'autre mais le sens des variations est irrégulier. La moyenne des données se situe aux environs de I5/mn au cours du bilan I et de $20 / \mathrm{mn}$ au cours du bilan II.

- En alimentation $D$, les données obtenues consécutivement au repas $H N$ sont toujours plus faibles qu'après le repas $\mathrm{BN}$. Le pourcentage de chute des fréquences respiratoires entre les 2 journées s'établit pour les $2^{\mathrm{e}}, 3^{\mathrm{e}}$ et $4^{\mathrm{e}}$ heures à I $9 \pm 4, \mathrm{II} \pm 3 \mathrm{et}$ $9 \mp 4$ pour le bilan I et à $20 \mp 2,20 \mp 7$ et $25 \mp 2$ pour le bilan II.

\section{Évolution de l'élimination urinaire d'azote consécutivement aux deux types de repas}

La collecte des émissions urinaires deux fois par jour et leur analyse étant difficile à mettre en cuvre sur les animaux en bilan, nous avons préalablement effectué cette étude sur un autre porc. L'animal de $53 \mathrm{~kg}$ est soumis au régime $\mathrm{D}:$ la teneur 
en $\mathrm{N}$ des repas $\mathrm{BN}$ et HN est respectivement de 0,82 et $8,0 \mathrm{p}$. Ioo de la matière sèche et les repas apportent soit $5,8 \mathrm{~g}$ soit $58,6 \mathrm{~g} \mathrm{~N}$. Par suite d'inappétence, son niveau journalier moyen de consommation est plus faible que celui des animaux en bilan ; il atteint respectivement pour la matière sèche et pour l'azote 75 et $1,32 \mathrm{~g} / \mathrm{P}^{0,75}$.

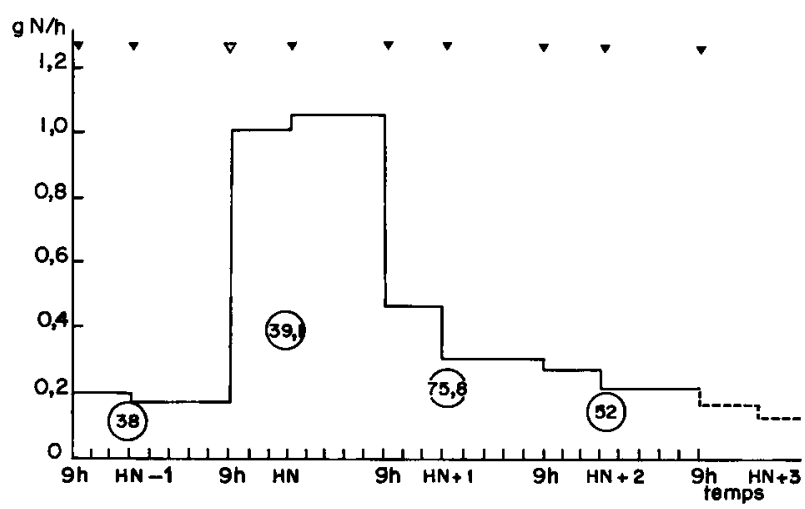

FIG. I. - Evolution de l'emission horaire d'azote urinaire en fonction des ingestions d'azote (moyenne!4 données)

$\begin{array}{llllll}\nabla & 5,83 & \nabla & 58,59 & \text { g Ni/repas } & \text { (38) Nur p. } 100 \mathrm{Ni}\end{array}$

La figure I montre l'évolution de l'émission horaire d'azote urinaire et précise le rapport $\mathrm{N}_{u r} / \mathrm{N}_{i}$ jours après jours (jours $\mathrm{HN}, \mathrm{HN}+\mathrm{I}, \mathrm{HN}+2, \mathrm{HN}+3=\mathrm{HN}-\mathrm{I}$ ). Elle confirme les observations faites précédemment : cette émission augmente très rapidement dans les heures qui suivent l'ingestion du repas HN (CHARLET-LERY, I970; WIESEMULLER et POPPE, I968). Malgré cette augmentation, le rapport $\mathrm{N}_{u r} / \mathrm{N}_{\mathrm{t}}$ ne varie pas 38-39,I, que l'animal ait ingéré dans la journée II,6 ou 64,4 g d'N (Cette même constance du rapport, 42-44, a été observée sur un autre animal ingérant soit 57 soit $87 \mathrm{~g} \mathrm{~N}$ par jour). Par contre, les jours $\mathrm{HN}+\mathrm{I}$ et $\mathrm{HN}+2$, ce rapport est très élevé 76 et 52 , mais diminue jusqu'à la valeur $3^{8}$ le jour $\mathrm{HN}+3$.

Prenant comme valeur minimum d'élimination celle du jour $\mathrm{HN}+3$ (ou $\mathrm{HN}-\mathrm{I}$ ), les $52,8 \mathrm{~g}$ d'azote supplémentaire du repas $\mathrm{HN}$ entraînent dans les 3 jours $\mathrm{HN}, \mathrm{HN}+\mathrm{I}, \mathrm{HN}+2$, un supplément urinaire de $26,6 \mathrm{~g}$, soit un rapport global $\mathrm{N}_{\text {ur }} / \mathrm{N}_{1}$ de 50 p. Ioo.

\section{DISCUSSION}

\section{Critique de la méthode}

Il est évident, à partir des résultats du tableau 4, que les bilans ont été prolongés d'une façon inefficace. Une durée de deux semaines conduit à des résultats pratiquement équivalents à ceux obtenus avec trois semaines pour le bilan I effectué avec 3 animaux. Des variations d'environ $\mathrm{I}$ point s'observent dans le bilan II où il n'y a 
que 2 animaux.'La réduction de la durée de 1'expérience à une seule semaine n'aurait pas modifié l'aspect comparatif des résultats, mais assuré moins de précision aux valeurs, de grosses variations existant d'une semaine sur l'autre.

\section{Validité des résultats}

Pour les animaux de $40 \mathrm{~kg}$, nos données (bilan : I7,39 g, CR : 60) sont très proches de celles obtenues par divers auteurs avec des animaux de poids d'âge et de croissance comparables, pour des protéines de haute valeur biologique (farine de hareng (DELORT-LAVAL et ZELTER I963), tourteau de soja (DELORT-LAVAL et BozALOPEZ, I964), poudre de lait écrémé et orge ('THORBECK, I967), tourteau de soja, farine de hareng et orge (OSLAGE et al., I966). Pour ces derniers auteurs, un apport d'azote excédentaire par rapport aux besoins des animaux ne modifie pas le bilan mais abaisse le CR à 40 (tabl. 7).

TABLEAU 7

Données comparées

\begin{tabular}{|c|c|c|c|c|c|c|}
\hline Auteurs & $\begin{array}{c}\text { Poids } \\
(\mathbf{k g})\end{array}$ & $\underset{(g / j)}{\text { Gain }}$ & $\begin{array}{c}\mathrm{MS}_{\mathrm{i}} \\
(\mathrm{g})\end{array}$ & $\begin{array}{c}\mathrm{N} \text { bilan } \\
\text { (g) }\end{array}$ & $\begin{array}{c}\mathrm{N}_{\mathrm{d}} / \mathrm{MOO}_{\mathrm{d}} \\
(\%)\end{array}$ & $\begin{array}{l}\text { Protéines/ } \\
\mathrm{kg} \text { gain }(\mathrm{g})\end{array}$ \\
\hline ThORBECK (1967). & 37 & 510 & 1130 & 16,4 & 29,6 & 201 \\
\hline OsLAGE $(1966) \ldots \ldots \ldots$ & 40 & - & 1770 & 17,2 & - & - \\
\hline Charlet-Lerty $\ldots \ldots \ldots$ & 40 & 560 & 1435 & 17,3 & 26,5 & 193 \\
\hline \multirow[t]{2}{*}{ THORBECK (1967) ....... } & $74(\mathrm{E})$ & 720 & 2160 & 16,9 & 20,7 & 147 \\
\hline & 73 (DF) & 800 & 2205 & 21,5 & 25,0 & 170 \\
\hline OsLaGE $(1966) \ldots$ & 75 & - & 2646 & 17,3 & - & - \\
\hline \multirow[t]{2}{*}{ Cunningham (1968) } & 73 & 596 & 2000 & 17,7 & - & 169 \\
\hline & 73 & 646 & 2000 & 19,1 & - & 185 \\
\hline \multirow[t]{2}{*}{ KORNEGAY (1968) } & 70 & 1090 & 2720 & 24,3 & - & 140 \\
\hline & 70 & 1250 & 2700 & 26,1 & 一 & 130 \\
\hline Delort-Laval (1964) & 74 & 900 & 2490 & 29,2 & 25,0 & 202 \\
\hline Charlet-Leryy $\ldots \ldots \ldots$ & 74 & 915 & 2490 & 25,2 & 22,1 & 172 \\
\hline
\end{tabular}

II. - Croissance

\begin{tabular}{|c|c|c|c|c|}
\hline Auteurs & $\begin{array}{c}\text { Phase } \\
\text { de croissance }(\mathrm{kg})\end{array}$ & Méthode & Gain & Protéines/kg gain \\
\hline $\begin{array}{l}\text { KOTARBINSKa et } \\
\text { KIELANOSKI }(1967) \ldots \\
\text { THORBECK }(1967) \ldots \ldots \\
\text { CHARLET-LERY } \ldots \ldots \ldots\end{array}$ & $\begin{array}{l}30-88 \\
30-85 \\
26-97\end{array}$ & $\begin{array}{c}\text { Abattage } \\
\text { Calcul à partir } \\
\text { gain de poids } \\
\text { id. }\end{array}$ & $\begin{array}{l}660 \\
655(1) \\
630\left(^{(2)}\right. \\
660\end{array}$ & $\begin{array}{l}155 \\
170 \\
157 \\
180\end{array}$ \\
\hline
\end{tabular}

(1) Animal E.

(2) Animaux D et F. 
Pour les sujets de $75 \mathrm{~kg}$, il serait erroné d'établir des comparaisons entre les données brutes car elles correspondent à des croissances très différentes. Par contre, le calcul de la quantité de protéines retenues par $\mathrm{kg}$ de gain, plus justifié, conduit à des valeurs très variables (I30 à $202 \mathrm{~g}$ ) qu'expliquent des conditions alimentaires - énergétiques et azotées - très différentes (tabl. 7).

Si l'on considère les expériences qui permettent soit la mesure réelle des protéines fixées par $\mathrm{kg}$ de gain (KotAARBINSKA et KrELANOSKI, I967) soit leur appréciation à partir de plusieurs bilans d'azote (THORBECK, I967, et nous-mêmes), sur des phases de croissance parfaitement comparables, on obtient des valeurs beaucoup plus homogènes, respectivement : I55, I70 et I57, I80. On peut admettre que les techniques utilisées expliquent, en partie, ces variations. Mais les différences individuelles que ces auteurs et nous-mêmes avons observées, laissent supposer qu'il pourrait aussi exister des différences raciales. Nos porcs de race Large White sont issus d'un troupeau dont les verrats sont testés sur la descendance pour la qualité de leur carcasse.

\section{Baisse de fréquence respiratoire}

Comme dans les expériences précédentes qui nous ont fait penser à ce protocole, on constate l'abaissement de fréquence respiratoire à la suite des repas HN par rapport aux repas BN. Ceci nous prouve d'une façon presque absolue que s'il nous avait été possible de mesurer les échanges respiratoires des animaux, nous aurions bien observé des dépenses postprandiales plus faibles après les repas HN qu'après les repas $\mathrm{BN}$ et que l'ensemble des réactions des animaux restent bien coordonnées.

\section{Opposition entre nos données et celles de la bibliographie}

Les raisons qui nous font aborder l'étude de la dissociation des apports énergétiques et protéiques chez le Porc et qui sont exposées dans l'introduction, sont éloignées de celles exprimées par les quelques auteurs qui ont étudié le même sujet.

Depuis de longues années, de nombreux auteurs ont démontré la nécessité d'un apport calorique suffisant qui, évitant l'utilisation des protéines à des fins énergétiques, permet d'obtenir une rétention d'azote importante. Mais l'obligation de faire rigoureusement coïncider dans le temps les apports énergétiques et protéiques est controversée. Les modalités de la dissociation sont d'ailleurs très diverses.

Sur les monogastriques adultes autres que le Porc, les auteurs observent le rôle favorable des glucides sur l'utilisation des protides et concluent à la supériorité de la mixité des apports (MUNRO, I949; GEIGER et al., I950) ou limitent la durée qui sépare l'ingestion de ces nutriments à $5 \mathrm{~h} \mathrm{I/2}$ chez 1'Homme à I2 heures chez le Rat et le Chien (MunRo et WIKRamanayake, I953).

Chez le Rat en croissance, l'alternance de semaines protéiprives et protéiques (MORIN-JomaIN, I963) ralentit très nettement la croissance des animaux par rapport à celle des sujets recevant régulièrement le régime protéique : les pertes d'azote subies en semaine protéiprive n'étant pas compensées par les bilans d'azote pourtant très élevés observés en semaine protéique. Mais si les ingestions de matière sèche et d'azote sont égalisées ou à peu près dans les deux lots par abaissement du taux protéique dans le régime témoin, la fixation d'azote corporel est peu modifiée : I9 $\mathrm{g}$ contre $2 \mathrm{I}, 8$ soit 17,44 et $\mathrm{I}$ 7, II $\mathrm{g}$ de protides par Ioo $\mathrm{g}$ de gain de poids (MoRIN JOMAIN, I969). 
EGGERT et al. (I953) sont les premiers à choisir comme animal d'expérience le Porc en croissance $(20-30 \mathrm{~kg})$ et à utiliser non pas des nutriments purs (glucose, caséine), mais des aliments complexes (céréales, tourteaux, farines animales). L'espacement des repas azotés toutes les 24 heures, ne modifie ni la croissance, ni les coefficients de digestibilité ( $80 \mathrm{p}$. I00) ni ceux de rétention ( $52 \mathrm{p}$. I00), ce que retrouvent YEO et CHAMBERLAIN dès I966, que confirment MENKE et al. (I969) et que laissent supposer les résultats de HENRY (Ig68). Les espacements atteignant 36 et 48 heures ne modifient pas les CUD, ralentissent significativement la croissance et abaissent les CR respectivement de 4 points non significativement, de 7 points significativement Les longues alternances ( 18,21 et 35 jours) de déplétion (50 p. roo des besoins) et de réplétion azotée ( $150 \mathrm{p}$. Ioo des besoins) ralentissent aussi la croissance (PENZES et MENTIER, Ig68).

Il y a à première vue, contradiction flagrante entre les résultats d'EGGERT et al., et les nôtres. Mais les deux expériences se différencient pas plusieurs points :

- par la rétention des protéines des régimes mixtes moindre pour EGGERT et al. (48-52 pour des porcs de $30-35 \mathrm{~kg}$ ) que pour nous (6o pour des pores de $40 \mathrm{~kg}$ ) avec. des teneurs des régimes en protéines voisines I36-I28 contre I28 g/ $/ \mathrm{kg}$;

- par la qualité des protéines des régimes BN, faible d'un côté (maïs seul) élevée de l'autre (céréales, farine de poisson et luzerne déshydratée) ;

- par la distribution des apports minéraux et vitaminiques aux seuls repas HN (EGGERT et al.) ou à tous les repas quels qu'ils soient (nous-mêmes) ;

- par l'espacement entre les repas HN. Nos espacements plus longs conduisent lors de l'ingestion de ces repas à un apport brutal d'azote dans l'organisme qui atteint par unité de poids métabolique 2,10 et $2,52 \mathrm{~g}$ respectivement pour les bilans I et II, soit plus de $30 \mathrm{~g}$ pour les animaux de $40 \mathrm{~kg}$ et plus de $60 \mathrm{~g}$ pour ceux de $70 \mathrm{~kg}$. L'espacement maximum d'EGGERT et al. (48 h) n'entraîne qu'un supplément d'ingestion d'environ $\mathrm{I}, 7 \mathrm{~g} / \mathrm{P}^{\mathbf{0}, 75}$.

Cette dernière observation met bien à nouveau en évidence l'existence d'un choc protéique dont nous avons déjà supposé la possibilité (CHARLET-LERY, I970), choc protéique dont les manifestations n'auraient lieu que pour des ingestions supplémentaires d'azote très élevées. Mais l'existence probable de ce choc ne permet pas d'expliquer les modalités de rétention des protéines des repas HN.

\section{Possibilité de stockage d'azote du repas $H N$}

Les constatations faites sur les animaux soumis aux études énergétiques nous avaient montré, comme nous le rappelons à la page 76 , que dans les 24 heures qui suivent le repas $\mathrm{HN}$, de 30 à $80 \mathrm{p}$. Ioo de $\mathrm{N}_{\mathbf{i}}+$ reste apparemment retenu par l'organisme. Mais il ne nous avait pas été alors possible de prolonger les collectes d'urine.

Les résultats des bilans d'azote des animaux soumis aux régimes classique ou dissocié, nous assurent que cet azote est utilisé dans les mêmes proportions que si son ingestion est régulière à tous les repas.

L'évolution de l'élimination de l'azote urinaire après l'ingestion du repas $\mathrm{HN}$ étudiée séparément mais dans des conditions voisines $\left(\mathrm{g} \mathrm{N}_{\mathbf{t}}+/ \mathrm{P}^{\mathbf{0}, 75}=2,69\right)$ permet de formuler une hypothèse sur les modalités de cette rétention. Bien qu'aucune mesure n'ait été faite sur l'élimination d'azote fécal jour après jour, il semble logique d'après tous les travaux déjà cités ici d'admettre que le CUD N est constant et on 
peut fixer sa valeur aux environs de 8o. Dans ces conditions, l'azote digestible à la disposition de l'animal est, selon les repas, de 4,66 ou de 46,9 et selon les jours de 9,3 ou de 5I,6. L'azote urinaire éliminé successivement les jours $\mathrm{HN}-\mathrm{I}, \mathrm{HN}$, $\mathrm{HN}+\mathrm{I}$, et $\mathrm{HN}+2$ étant de $4,4 \mathrm{~g}, 23,8 \mathrm{~g}, 8,9 \mathrm{~g}$ et $6, \mathrm{I} \mathrm{g}$, on pourrait calculer les CR suivants : 53, 54,4 et 34. L'efficacité très faible de l'azote les jours $\mathrm{HN}+\mathrm{I}$ et $\mathrm{HN}+2$ à un moment où l'azote est ingéré en faible quantité apparaît improbable. Il serait donc plus logique de penser que l'N du repas HN retenu par l'organisme animal sous une forme difficile à préciser (tissus intestinaux, foie,...), mais labile, serait progressivement utilisé par l'animal et donnerait lieu alors à la formation et à l'élimination d'azote urinaire. Dans cette optique, les 42,2 $\mathrm{g}$ d'azote digestible $\mathrm{du}$ repas HN auraient provoqué un supplément d'azote urinaire de $26,6 \mathrm{~g}$, leur CR sera de $40 \mathrm{p}$. Ioo, et au cours des 4 jours consécutifs, on pourrait calculer un CR global de $1^{\prime}$ azote de 46 p. Ioo (1)

Cependant, le stockage de 72 et 96 heures dont nous devons faire l'hypothèse, sans en préciser le lieu, dépasse de beaucoup les possibilités qui ont été envisagées jusqu'à ce jour. Par contre, de nombreux auteurs ont montré la rapidité de modifications de certains systèmes enzymatiques lors des changements de régimes, entre autres SzEPECI et FREELAND (I968).

On peut rapprocher nos résultats de ceux de CALET (I968) sur Poulet en croissance. Lorsque cet animal dispose en libre choix d'aliments protéique et protéiprive, il ingère en une seule fois et rapidement ses protéines. Il y a donc là, dissociation dans le temps dont l'importance relative doit être appréciée en considérant que la vitesse de transit intestinal est beaucoup plus rapide chez la volaille que chez le Porc. Comme dans notre cas, elle ne provoque pas de modification de l'utilisation métabolique de l'azote.

\section{Croissance et caractéristiques des carcasses}

Les vitesses de croissance et les caractéristiques des carcasses sont très voisines. La tendance à la moindre adiposité des carcasses correspond bien à l'hypothèse émise en commençant ce travail. L'ampleur de ce phénomène est faible mais théoriquement la limitation de la lipogenèse ne pouvait dépasser un pourcentage égal à : nombre d'heures de lipolyse consécutives aux 2 repas $\mathrm{HN} /$ nombre d'heures de la semaine, soit 8 à $9 \mathrm{p}$. Ioo, différence qui nécessite pour être mise en évidence, un nombre d'animaux beaucoup plus important que le nôtre.

Par contre, il ne semble pas comme nous en avons émis l'hypothèse que l'alimentation $\mathrm{D}$ ait provoqué un abaissement des dépenses caloriques puisque, à niveau égal d'ingestion avec l'alimentation $C$, les indices de consommation sont légèrement, mais non significativement plus élevés $2,96 \mp 0,12$ contre $2,8 \mathrm{I} \mp 0,2 \mathrm{I}$. L'hypothèse d'une moindre dépense d'entretien avec les régimes pauvres en azote a été vérifiée sur les animaux en régime $\mathrm{C}$. Or, en régime $\mathrm{D}$, il n'est pas impossible que le catabolisme azote supplémentaire observé les jours $\mathrm{HN}+\mathrm{I}$ et $\mathrm{HN}+2$ ait provoqué des dépenses d'énergie plus importantes que le laissent prévoir les faibles ingestions d'azote correspondantes.

Les valeurs obtenues au cours des périodes de bilan : rétention d'azote et crois-

(1) Bien que les valeurs soient proches, la comparaison avec les bilans de 3 semaines ne peut être faite p uisque les conditions alimentaires sont légèrement différentes. 
sance pondérale permettent de calculer les quantités de protéines fixées par $\mathrm{kg}$ de gain. Pour les sujets témoin 3 et 5 et ceux en alimentation $D 9$ et II, on obtient les valeurs suivantes : bilan I : $2 I_{4}$ et 2 II contre 222 et 202 , bilan II : I 70 et I 74 contre I76 et I67. Ceci confirme que la protéinogenèse diminue avec 1'âge de 1'animal d'environ I $8 \mathrm{p}$. Ioo entre 40 et $75 \mathrm{~kg}$ et montre qu'elle n'a pas été modifiée par le type de régime.

La comparaison entre résultats de bilan et de croissance totale est surtout intéressante lorsqu'on considère les animaux pris individuellement. Le couple (3-9) ayant les indices de consommation les plus bas $(2,74$ et $2,8 \mathrm{I})$ présentent les CR les plus élevés (bilan I : 63,3 et 65 , I, bilan II : 59,8 et 59,4) et les meilleures caractéristiques de carcasses (p. Ioo jambon : 55,3 ; p. Ioo bardière : 15,8 et $\mathrm{I}_{4,8}$; épaisseur lard : $22,5$ et $2 \mathrm{I}, 0)$. L,e couple (5-II) a inversement des indices de consommation plus élevés (3,I4 et 3,05), des CR plus bas (bilan I : 54,6 et $6 \mathrm{I}, 4$; bilan II : $5 \mathrm{I}, 9$ et $53, \mathrm{I}$ ) et des caractéristiques de carcasse moins bonnes (p. Ioo jambon 48,4 et 52,8 ; bardière 22,8 et I8,9; épaisseur de lard : 30 et 26 ).

Cet ensemble de valeurs parfaitement logiques met nettement en évidence l'importance du facteur génétique comme nous l'avons déjà signalé.

\title{
CONCLUSIONS
}

Le fait que la dissociation partielle des apports énergétiques et azotés permette les mêmes fixations d'azote corporelles et les mêmes croissances que leur association, doit conduire à ne pas condamner les méthodes qui se basent sur cette connaissance et à les étudier du point de vue économique. En effet, même si la supplémentation protéique bi-hebdomadaire ne conduit qu'à une amélioration très faible dela qualité des carcasses produites, elle peut alléger la charge économique de la main-d'œuvre dans l'alimentation. Mais l'application de nos résultats à des fins pratiques nécessite des transpositions qui seront d'autant plus faciles à concevoir que l'on aura précisé les modifications métaboliques induites par les repas $H N$, études que nous continuons par ailleurs. Cependant, les résultats de Rotermel (I96o) font supposer qu'une telle méthode serait applicable au plan de la pratique.

Reçu pourpublication en octobre 1970.

\section{SUMMARY}

\author{
EFFECT OF GIVING PROTEIN SUPPLEMENT TWICE A WEEK \\ WITH A DIET LOW IN NITROGEN ON WEIGHT GAIN \\ AND NITROGEN BALANCES IN GROWING PIGS
}

Growing pigs from 26 to $98 \mathrm{~kg}$ were given the same amount of energy and protein per week. For one group the feed was given in the usual way, in I 3 equal feeds. The other got II feeds poor in $\mathrm{N}$ (BN) and 2 rich in $\mathrm{N}$ (HN) separated by 72 or $96 \mathrm{~h}$, termed separate feeding.

Growth was similar in the 2 groups, 677 and $644 \mathrm{~g}$ daily, and intakes per kg gained also were close, $2.8 \mathrm{I}$ and $2.96 \mathrm{~kg}$. There was also no difference in $\mathrm{N}$ balance estimated for $2 \mathrm{I}$ consecutive 
days at about $4^{\circ} \mathrm{kg}$ and again at $70 \mathrm{~kg}$ liveweight. At the lower weight, daily retentions were 17.2 and $17.5 \mathrm{~g}$ and at the higher weight 24.9 and $25.5 \mathrm{~g}$, giving retentions of 60 and $56 \mathrm{p}$. Ioo at the respective weights.

Carcasses of the pigs on separate feeding tented to be less fat. It may be assumed that the animal would temporarily store the protein given to it irregularly and would use it on the 2 following days.

\section{RÉFÉRENCES BIBLIOGRAPHIQUES}

Calet C., I968. Quelques conséquences de J'alimentation azotée du poussin. World Rev. anim. Prod., 4, $77-86$.

Charlet-LeRY G., r96o. Importance des dépenses postprandiales selon le type de rationnement chez le Porc et influence de ce dernier sur le métabolisme de base approché. Proc. VIİ Congr. Int. Nut., 5, 23-30.

Charlet-LeRY G., 1967. Influence of the dissociation of energy and protein feeding on energetic efficiency in growing pigs. Proc. 4th symp on energy metab. of farm anim., 275-28r. Priel Press Id. Newcastle.

Charlet-Lery G., Zelter S. Z., ig69. Efficacité de quelques protides alimentaires chez le Porc. VI. Farine de baleine déshydratée à haute température, tourteau d'arachide et farine de hareng. Ann. Zootechn., 18, 33-40.

Charlet-Lery G., r97o. Variations d'extra-chaleur induites par l'ingestion de repas très riches en protéines chez le porc en croissance. Ann. Biol. anim. Bioch. Biophys., 10, 123-143.

Cunningham H. M., x968. Effect of caffeine on nitrogen retention, carcass composition, fat mobilization on the oxidation of $\mathrm{C}_{14}$ labeled body fat composition. J. Anim. Sci., 27, 424-430.

Delort-Laval J., Zelter S. Z., 1963. Effet de la conduite du séchage à la flamme sur la valeur biologique de farines de harengs chez le Porc. Ann. Zootech., 12, 193-202.

Delort-Iaval, J., Boza-Lopez J., 1964. Efficacité de quelques protides alimentaires chez le Porc. V. Influence du traitement technologique sur la valeur des protéines du tourteau de soja. Validité de quelques tests biochimiques pour l'appréciation de la qualité des tourteaux. Ann. Zootech. 13, 35-5o.

Eggert R. G., Brinegar M.-J., Anderson C. R., 1953. Delayed protein supplementation of corn diets for growing swine. J. Nutr., 50, 469-477.

Geiger E., Bancroft R. W., Hagerty E. B., 1950. The nitrogen sparing effect of dietary carbohydrate in its relation to the time factor experiments with repletion of protein-depleted adult rats. $J$. Nutr., 42, 577-685.

Henry Y., 1968. Libre consommation de principes énergétiques et azotés chez le Rat et chez le Porc selon la nature de la source azotée, sa concentration dans le régime et le mode de présentation. Ann. Nutr. Alim., 22, 12 I-140.

Kotarbinska M., Kielanowski J., I967. Long-Term calorimetric measurements on groups of growing pigs. Proc. 4th Symp. energy metab. farm. anim., 31 I-318. Oriel Press Ld. Newcastle.

Kornegay E. T., Graber G., I968. Effect of food intake and moisture content on weight gain, digestibility of diet constituents and N retention of surne. J. Anim. Sci., 27, I59I-95.

Menke K. H., Lantzsch H. J., Ehrensvard U., Schneider W., I969. Einfluss der alternierenden Fütterung von Getreide und Eiveissergänzungsfutter auf Verdaulichkeit, Stickstoff Bilanz und Blutharnstoffgehalt beim Schissein. Landrvirtsch. Forsch. Dts ch., 22, 173-181.

Morin-Jomain M., 1963. Le comportement nutritionnel du rat soumis alternativement à des régimes protéiprives et à des régimes protéiques. Arch. Sci. Physiol. Paris, 17, 55-74.

Morin-Jomain M., I969. Composition corporelle, glycogène hépatique et glycémie du rat blanc soumis à deux types d'alimentation discontinue : jeûne total ou protéique intermittent. Arch. Soc. Physiol., 23, $21-45$.

Munro H. N., 1949. The relationship of carbohydrate metabolism to protein metabolism. III. Furthers observations on time of carbohydrate ingestion as a factor in protein utilization by the adult rat. $J$. Nutr., 89, 375-391.

Munro H. N., Wikramanayake T. W., 1954. Absence of a time factor in the relationship between level of energy intake and protein metabolism. $J$. Nutr., 52, 99-II4.

Oslage H.-J., Fliegel H., Farries F. E., RichteR K., I966. Stickstoff-Fett und Energieansatz bei wachsenden Mastschweinen. Zeitsch. Tierphys. Tierernähr. Fùttermit., 21, 50-65.

Penzes L., Mentler L., I968. Effet d'une alimentation alternée avec des régimes riches ou pauvres en protéines sur le gain de poids et les fractions protéiques du sang chez le Porc en croissance. Acta agron. hung., 17, 195-204.

Rotermel Z. A., 196o. Influence d'une méthode alternée d'alimentation sur l'avancement de la maturité et les résultats d'engraissement avec des porcs Lavge White purs. Engraissement des porcs avec une alimentation alternée. Ucen. Zap. Kazan vet. Inst., 1960, 77, 23-33, 43-49. In N.A.R 1962, 32, n 6394 et 6395 . 
SzePeCI B., Freedland R. A., Ig68. Alterations in the activities of several rat liver enzymes at various times after the feeding of high carbohydrates diets to rats previously adapted to a high protein regimen. J. Nutr., 84, 37-46.

Tноввеск G., I967. Studies on energy metabolism of growing pigs. Ath Symp. Energy metab. (distributed paper).

Wiesemuller W., Poppe S., ig68. Die Bewertung der proteine durch die Bestimmung der N-Bilanz. II. Untersuchungen über den Zeitpunkt des Maximums der N-Ausscheidung aus Kot und Harn nach der Futterung bei Ratte und Schwein. Arch. Tierernäh., 18, 474-483.

Yeo M. L., Chamberlain A. G., I 966 . Delayed protein supplementation of barley diets for weanling pigs. Proc. Nutr. Soc., 25, XII. 